\title{
El rol del gluten en la panificación y el desafío de prescindir de su aporte en la elaboración de pan
}

\author{
Sciarini L.S.; M.E. Steffolani y A.E. León
}

\begin{abstract}
RESUMEN
La enfermedad celíaca es una enteropatía crónica del intestino delgado inmunomediada y promovida por la exposición a una dieta con gluten en individuos genéticamente predispuestos. La interacción de factores genéticos y ambientales provoca la pérdida de tolerancia a ciertas proteínas presentes en algunos cereales. Recientemente se estableció una prevalencia de celiaquía del 1\% en el mundo, con grandes variaciones entre los países. El único tratamiento eficaz es adoptar una dieta libre de gluten. El gluten es una red proteica que se estructura al hidratar la harina y se refuerza mediante el amasado. Está conformado fundamentalmente por dos fracciones proteicas: prolaminas y glutelinas. Estas proteínas juegan un rol preponderante en la calidad de los más tradicionales productos de panificación, por lo que es un gran desafío para investigadores y tecnólogos elaborar estos productos sin contar con esta red. La polimerización de proteínas para simular la red de gluten ha mostrado sus limitaciones, por lo que se profundizó en el estudio de las interacciones entre diferentes componentes como un factor determinante para el desarrollo de productos. Sobre esa base se obtuvieron mejores resultados usando almidón de mandioca y harina de soja activa, gracias a las interacciones específicas establecidas entre ambos componentes.
\end{abstract}

Palabras clave: gluten, enfermedad celíaca, prolaminas, libres de gluten, panificación.

Sciarini L.S.; M.E. Steffolani and A.E. León, 2016. The role of gluten in breadmaking and the challenge of elaborating gluten-free bread. Agriscientia 33 (2): 61-74

\section{SUMMARY}

Celiac disease is an immunomodulated chronic enteropathy triggered by gluten ingestion in genetically susceptible patients. The interaction between genetic 
and environmental factors leads to an intolerance toward some proteins present in certain cereals. Recently, the worldwide prevalence of this disease has been estimated in 1\%, with an important variation among different countries. Nowadays, the only effective treatment for the disease consists in a lifelong gluten-free diet. Gluten is a protein network formed when flour and water are mixed (energy input), and is composed of two main protein fractions: prolamins and glutenins. This protein network is a leading factor determining the quality of most traditional baked products; therefore, obtaining these products without this network is a major challenge for food scientists and technologists. Protein polymerization -other than gluten- has shown limited results in replacing the gluten network; hence, a different approach was adopted, focusing on the study of interactions among different raw components as a key factor in glutenfree products development. With this approach, better results were obtained when using cassava starch and active soy flour, due to specific interactions established between both components.

Key words: gluten, celiac disease, prolamins, gluten-free, breadmaking.

L.S. Sciarini: Instituto de Ciencia y Tecnología de los Alimentos Córdoba (ICYTAC), CONICET-UNC. M.E. Steffolani y A.E. León: ICYTAC, CONICET-UNC, Facultad de Ciencias Agropecuarias, Universidad Nacional de Córdoba, CC 509, 5000, Córdoba, Argentina. Correspondencia a: aeleon@agro.unc.edu.ar

\section{INTRODUCCIÓN}

Las proteínas de los cereales se clasifican de acuerdo a su solubilidad en cuatro tipos (Osborne, 1907):

- Albúminas: solubles en agua.

- Globulinas: insolubles en agua y solubles en soluciones salinas diluidas.

- Prolaminas: insolubles en agua y en soluciones salinas y solubles en alcohol al $70 \%$.

- Glutelinas: insolubles en los solventes anteriormente mencionados y solubles en ácidos o bases diluidos o en detergentes.

Esta clasificación no refleja relaciones genéticas o químicas de los polipéptidos que componen cada fracción (Tatham et al., 1985); sin embargo, es la más ampliamente difundida hasta el momento.

A los dos primeros grupos pertenecen las proteínas metabólicamente activas que se ubican en el citoplasma celular; las otras sirven como fuente de nitrógeno durante la germinación de la semilla.

En el trigo las prolaminas y las glutelinas se denominan gliadinas y gluteninas, respectivamente; estas fracciones son las que conforman el gluten y constituyen alrededor de $75-80 \%$ del total de proteínas. Las albúminas y globulinas son proteínas no formadoras de gluten y representan $20-25 \%$ del total. Las proteínas formadoras de gluten juegan un papel fundamental en la elaboración de produc- tos de panificación, fundamentalmente en aquellos que son sometidos a un proceso de leudado.

Por otra parte, la ingesta de gluten, aun en pequeñas cantidades, puede producir una serie de trastornos en algunas personas, entre los que se incluyen la dermatitis herpetiforme, la ataxia por gluten, la sensibilidad al gluten no celíaca y la enfermedad celiaca (EC) (Ludvigsson et al., 2013).

En el presente trabajo se revisa el rol que desempeña el gluten en la obtención de productos leudados y las dificultades para sustituirlo en la elaboración de panes libres de gluten. Al mismo tiempo, se analizan los diferentes enfoques aplicados y se profundiza en los resultados obtenidos sobre la base del estudio de las interacciones que se establecen entre los diferentes componentes de los ingredientes usados.

\section{ENFERMEDAD CELÍACA}

La EC es definida como una enteropatía crónica del intestino delgado inmuno-mediada y promovida por la exposición a una dieta con gluten en individuos genéticamente predispuestos. La interacción de factores genéticos y ambientales lleva a la pérdida de tolerancia a ciertas proteínas presentes en algunos cereales, particularmente a las prolaminas del trigo, cebada, centeno y especies híbridas como el triticale, y en algunos casos, de la avena.

La inflamación permanente de la mucosa intestinal conlleva al aplanamiento de las vellosidades, 
que deriva en la mala absorción de nutrientes. Los síntomas más típicos de la enfermedad incluyen diarrea, pérdida de peso, estrés, vómitos, anemia y dolor abdominal. El único tratamiento que existe en la actualidad es el apego a una dieta libre de gluten de por vida. Si esta dieta no se lleva a cabo adecuadamente, pueden desencadenarse complicaciones como osteoporosis, desarrollo de linfomas intestinales, o pueden presentarse problemas en el crecimiento y en la fertilidad de los pacientes.

\section{Epidemiología}

La prevalencia de una enfermedad es definida como el número de casos presentes en una población en un momento dado. En 1950 la enfermedad se diagnosticaba por síntomas gastrointestinales, estudios de absorción y atrofia de las vellosidades del intestino delgado mediante una biopsia; así se estimaba una prevalencia de 1 en 8000 en la población general (Davidson \& Fountain, 1950). Esta estimación empezó a crecer a partir de la aplicación de análisis serológicos que permitían seleccionar individuos asintomáticos o silentes, a quienes se les confirmaba la celiaquía por medio de una biopsia intestinal.

Mediante la aplicación de estudios serológicos se observó un claro incremento en la prevalencia y notorias diferencias entre las distintas regiones del mundo. Estudios realizados en donantes de sangre en Suecia mostraron una prevalencia de 1:256 (Grodzinsky et al., 1992). Recientemente en Alemania se estimó una prevalencia de 0,9\% en niños y adolescentes (Laass et al., 2015). Relevamientos de similares características indicaron los siguientes resultados: 1:250 en Estados Unidos de América (Not et al., 1998), 1:330 en Países Bajos (Rostami et al., 1999), 1:99 en Finlandia (Kolho et al., 1998) y 1:157 en Israel (Shamir et al., 2002). En Brasil, un estudio realizado en San Pablo mostró una prevalencia de 1:273, y otro en Brasilia, de 1:681 (Gandolfi et al., 2000). En 1999, en la Argentina se efectuó un estudio poblacional sobre muestras de suero de exámenes prematrimoniales en el área de La Plata, que arrojó una prevalencia de 1:167 (Gómez et al., 2001), mientras que en un estudio con niños la prevalencia hallada fue de 1,26\% (1/79 niños) diagnosticados por histopatología con predominio del sexo femenino (Mora et al., 2012).

\section{Etiología}

La intolerancia al gluten se debe a una respuesta inadecuada del sistema inmune ante la exposi- ción a proteínas de las prolaminas en individuos genéticamente susceptibles. La etiología es multifactorial, con interrelaciones complejas de factores genéticos, inmunológicos y ambientales.

\section{Factores inmunológicos}

A fin de comprender las bases de la EC, es necesario conocer los mecanismos inmunológicos subyacentes. A tal fin, se expondrán sintéticamente algunos conceptos referentes al sistema inmunológico.

\section{Sistema inmune}

El funcionamiento del sistema inmune se divide en inmunidad innata e inmunidad adaptativa. La inmunidad innata constituye la primera línea de defensa inmunológica y se caracteriza por poseer mecanismos efectores, como fagocitos, complementos y péptidos antimicrobianos, que se activan en forma rápida y vigorosa frente a la presencia de un antígeno. Por su parte, la inmunidad adaptativa gira alrededor de los linfocitos $T$ y $B$, que poseen receptores altamente específicos y variados que se unen a los antígenos y disparan la activación y proliferación celular en un proceso conocido como expansión clonal. La expansión clonal de los linfocitos es necesaria para generar una respuesta inmunitaria eficiente, pero es lenta, ya que toma entre 3 a 5 días para generar linfocitos efectores. Por esta razón, la inmunidad innata es fundamental para controlar la mayoría de las infecciones o agresiones cutáneas y para disparar la inmunidad adaptativa (Medzhitov \& Janeway, 2000; Janeway, 2001).

El nexo entre la inmunidad innata y la adaptativa se debe a las células presentadoras de antígeno (CPA), que tienen como función captar, procesar y presentar moléculas antigénicas para que sean reconocidas luego por los linfocitos T y, así, dar comienzo a una respuesta inmune adecuada. Para este fin, las CPA poseen en sus membranas un grupo de receptores que forman parte del llamado complejo mayor de histocompatibilidad que, en humanos, recibe el nombre de antígeno leucocitario humano $(\mathrm{ALH})$.

\section{Factores genéticos}

Los enfermos celíacos se caracterizan por poseer un grupo de receptores de membrana ALH que son poco frecuentes en la población general; así, un alto porcentaje (95\%) de los pacientes celía- 
cos lleva en su dotación génica los alelos que codifican para el receptor ALH-DQ2, y el otro 5\% porta los genes que codifican para el receptor ALH-DQ8. Cabe destacar que el porcentaje de estos genes en la población general es de sólo un 40\%.

\section{Factores ambientales}

Las prolaminas del gluten poseen ciertas características que contribuyen a sus propiedades inmunogénicas. Son ricas en prolina y glutamina; debido al alto contenido en prolina, el gluten es resistente en gran parte a la degradación proteolítica en el tracto gastrointestinal, ya que las enzimas gástricas y pancreáticas no poseen capacidad proteolítica post-prolina.

Las porciones del gluten que son resistentes a la degradación enzimática son transportadas a través de la barrera epitelial como polipéptidos. Esos péptidos son modificados por la transglutaminasa tisular (TG2); ésta es una aciltransferasa que cataliza dos tipos de reacciones diferentes. Una de ellas es la transaminación, que es la formación de la unión isopeptídica entre residuos de lisina y glutamina (Seguro et al., 1996). En ausencia de aminas primarias ocurre la deaminación, donde el agua actúa como aceptora de grupos acilo, y la glutamina es entonces deaminada y transformada en ácido glutámico, cargado negativamente. Las moléculas ALH-DQ2 y -DQ8 de las CPA tienen preferencia por los aminoácidos cargados negativamente, por lo que se unen con mayor afinidad a los péptidos del gluten deaminados. De esta manera, las CPA se unen a estos péptidos, presentándolos como antígenos a los linfocitos B, y dando comienzo a una cascada de reacciones inmunológicas que tienen como resultado la producción de anticuerpos anti-gliadina, anti-TG2, y un ataque a los enterocitos. Este ataque produce el aplanamiento de las vellosidades intestinales, desfavoreciendo la correcta función absortiva del intestino.

\section{REQUERIMIENTOS PARA ALIMENTOS LIBRES DE GLUTEN}

El Codex Alimentarius (2008) establece que los alimentos exentos de gluten son aquellos que están constituidos o elaborados únicamente con uno o más ingredientes que no contienen trigo (es decir, todas las especies de Triticum, incluyendo el trigo, la espelta y el kamut), centeno, cebada, avena (aunque la avena puede ser tolerada por algunos celíacos) o sus variedades híbridas, y cuyo contenido de gluten no sobrepase los $20 \mathrm{mg} / \mathrm{kg}$ en total, medido en los alimentos tal como se venden o distribuyen al consumidor; o alimentos que estén constituidos por estos ingredientes, pero que hayan sido procesados para eliminar el gluten, y en los que el contenido de éste no sobrepase los 20 $\mathrm{mg} / \mathrm{kg}$ en total. Además, establece que los productos que sustituyan a alimentos básicos importantes deberían suministrar aproximadamente la misma cantidad de vitaminas y minerales que los alimentos originales a los que sustituyen.

Para comprender la importancia de la ausencia de gluten en los productos para celíacos, es necesario revisar el rol que el gluten tiene en la elaboración de pan.

\section{ROL DEL GLUTEN EN LOS PRODUCTOS DE PANIFICACIÓN}

\section{Pan}

El pan es un alimento nutricionalmente balanceado ya que es fuente de energía, proteínas, vitaminas (en particular del complejo vitamínico B), minerales y fibra dietética. En la Argentina, el $71,3 \%$ del trigo destinado al mercado interno en el año 2006 se empleó para la fabricación de pan, y el consumo per cápita/año de este producto en el mismo período fue de 72,5 kg/habitante/año (Lezcano, 2006).

Es un producto leudado obtenido gracias a la acción de levaduras que fermentan el azúcar de la harina de trigo producida por la hidrólisis del almidón (por parte de las enzimas naturalmente presentes en la harina). Durante la fermentación se generan gases como producto de la actividad metabólica de las levaduras. Muchos microorganismos pueden fermentar azúcares para producir dióxido de carbono $\left(\mathrm{CO}_{2}\right)$, pero el organismo más eficiente a tal fin en la masa panaria es Saccharomyces cerevisiae. Los principales productos de la fermentación son, en este caso, el $\mathrm{CO}_{2}$ y el alcohol etílico, resultado de una serie compleja de reacciones controladas por enzimas. $\mathrm{El} \mathrm{CO}_{2}$ es el responsable del aumento de volumen de la masa, mientras que el alcohol colabora en la producción del aroma complejo de estos productos horneados.

\section{Panificación}

Con el objeto de convertir la mezcla de ingredientes en una estructura esponjosa como lo es la miga de pan, se siguen una serie de procesos que llevan, en su conjunto, a la obtención de una masa con las propiedades mecánicas apropiadas que permitan la retención de gases, que resulta en 
una pieza de pan expandida con una estructura de miga pareja (Gan et al., 1990; Kokelaar \& Prins, 1995; McRitchie, 2016). Por medio de estos procesos se persiguen los siguientes objetivos: 1) mezclar y desarrollar la masa (por medio del amasado y la fermentación), 2) formar una estructura tipo espuma (por medio del moldeado y la fermentación), y 3) estabilizar la estructura porosa alterando la configuración molecular de los componentes poliméricos en las paredes de los alvéolos por medio de la aplicación de calor (horneado) (Kent \& Evers, 1994).

Independientemente de la manera en que se realicen el amasado y la fermentación, tres hechos ocurren fehacientemente (Bloksma, 1990; Campbell et al., 1991; Kent \& Evers, 1994): 1) Hidratación: el amasado de los ingredientes, además de la tarea obvia de mezclarlos, tiene como función distribuir el agua entre los polímeros. 2) Desarrollo del gluten: aunque algunas de las proteínas presentes en la harina son solubles en agua, es en las proteínas insolubles, conocidas colectivamente como gluten, donde se enfocan los estudios que pretenden elucidar cómo el trabajo aplicado transforma al agua y la harina en una masa cohesiva (Weegels et al., 1996). Cuando la masa se desarrolla bajo condiciones óptimas, estas proteínas forman una red viscoelástica que retiene el aire, y contiene a los gránulos de almidón y otros materiales, como el salvado. El óptimo desarrollo del gluten durante el amasado es vital para la formación de la estructura de la miga. 3) Incorporación y retención del aire: durante el amasado debe incorporarse el aire que formará la fase gaseosa de la miga de pan, y el volumen final de aire será una función de la amasadora que se emplee.

\section{Proteínas}

En 1948, Finney y Barmore encontraron una correlación positiva entre el contenido de proteínas de la harina de trigo y el volumen de pan. Posteriormente se observó que el volumen de pan depende en mayor medida de la calidad de las proteínas de la harina que de la cantidad en sí. La calidad proteica se refiere no sólo al tipo de proteína identificada según la clasificación de Osborne (1907), sino también a la influencia de cada fracción en la funcionalidad de la masa y, consecuentemente, en la calidad del pan. Con base en estos hechos, se utiliza más frecuentemente otra clasificación de las proteínas de la harina de trigo: proteínas que no forman gluten y proteínas formadoras de gluten.

Las proteínas que no forman gluten representan $15-20 \%$ del total de las proteínas del trigo; son solubles en soluciones salinas diluidas, por lo tanto corresponden a las albúminas y globulinas del fraccionamiento de Osborne (1907). Son proteínas monoméricas, metabólicamente activas y/o estructurales que se ubican en las capas más externas del grano de trigo. Su función en la panificación no ha sido claramente establecida (Veraverbeke \& Delcour, 2002). Estas proteínas poseen un buen balance de aminoácidos, contienen proporciones relativamente altas de lisina, triptofano y metionina, tres aminoácidos que se encuentran en baja proporción en la mayoría de los cereales.

Las proteínas formadoras de gluten se denominan también proteínas de almacenamiento, representan $80-85 \%$ del total de las proteínas del trigo y sirven como fuente de nitrógeno durante la germinación de la semilla ( $\mathrm{Ng} \&$ Bushuk, 1987; Larroque et al., 2000). Se encuentran en el endosperma del grano de trigo maduro, donde forman una matriz continua alrededor de los gránulos de almidón. Las proteínas del gluten son insolubles en agua o en soluciones salinas diluidas y pueden ser divididas en dos grupos de acuerdo a su funcionalidad: las gliadinas monoméricas y las gluteninas poliméricas. La composición de aminoácidos de las gliadinas y de las gluteninas es similar; se caracterizan por tener altas concentraciones de glutamina (un tercio o más del residuo total de aminoácidos) y de prolina (una séptima parte o más del residuo total de aminoácidos). En síntesis, se considera que las gliadinas son las responsables de la viscosidad del gluten, mientras que las gluteninas son las encargadas de conferir fuerza y elasticidad al gluten (Hoseney, 1994).

\section{Gliadinas}

Como se mencionó anteriormente, las gliadinas son mayormente proteínas monoméricas ricas en glutamina y prolina y, además, poseen un bajo nivel de aminoácidos cargados. Constituyen un tercio de las proteínas de la harina (Lindahl, 1990) y son clasificadas en $\alpha-, \beta-, \gamma$ - y $\omega$-gliadinas conforme a su movilidad en electroforesis a $\mathrm{pH}$ ácido (Shewry et al., 1986). Estas proteínas poseen una masa molecular entre $30 \mathrm{KDa}$ y $80 \mathrm{KDa}$ y están formadas por un dominio no repetitivo rico en estructura secundaria $\alpha$-hélice y por un dominio repetitivo heterogéneo rico en estructura giro- $\beta$ (Tatham \& Shewry, 1985). Las $\omega$-gliadinas no tienen residuos de cisteína, por lo que no pueden formar interacciones covalentes en la masa (Tatham, 1996). Las $\gamma$-gliadinas poseen ocho residuos de cisteínas que se encuentran localizados en el dominio C-terminal y forman cuatro enlaces disulfuro intramolecula- 
res, por lo que tampoco son capaces de formar enlaces covalentes con otras proteínas del gluten. Las $\alpha$-gliadinas poseen seis residuos de cisteínas igualmente ubicados como en las $\gamma$-gliadinas que forman tres enlaces disulfuro también intramoleculares. Sin embargo, estos enlaces disulfuro son importantes para retener el plegado de la estructura de las gliadinas que determina la naturaleza de las interacciones no covalentes con la red de gluteninas (Wrigley et al., 1998). Algunos investigadores proponen que son las responsables de la cohesividad de las masas (Hoseney, 1994), aunque otros sugieren que controlan el potencial de volumen de pan (Khatkar et al., 2002).

\section{Gluteninas}

Las gluteninas forman agregados proteicos unidos por puentes disulfuro y fuerzas no covalentes intermoleculares. La posibilidad de establecer enlaces covalentes entre diferentes moléculas es el rasgo distintivo de estas proteínas y explica sus elevadas masas moleculares, que varían desde 500 KDa a más de 10000 KDa (Wieser, 2007). Luego de la reducción de los puentes disulfuro, las subunidades de glutenina resultantes poseen una solubilidad en alcohol similar a las gliadinas y pueden ser divididas en dos tipos: las subunidades de alta masa molecular (HMW-GS) y las subunidades de baja masa molecular (LMW-GS) (Shewry et al., 1992). Las LMW- GS son un grupo complejo de proteínas que poseen la habilidad de formar enlaces disulfuro que permiten su incorporación dentro de los polímeros de gluteninas. La mayoría de las LMW-GS contienen entre siete y ocho residuos de cisteínas (Grosch \& Wieser, 1999; Wieser, 2003); seis residuos están en una posición homóloga a las gliadinas, y por lo tanto formarían puentes disulfuro intramoleculares. Los residuos de cisteínas adicionales no son capaces de formar puentes disulfuro intramoleculares, probablemente por impedimento estérico, por lo que establecerían puentes disulfuro intermoleculares formando agregados proteicos entre ellas y con HMW-GS (Pogna et al., 1994). Muy poco se conoce sobre la estructura de las LMW-GS. Tatham et al. (1987) propusieron que en el dominio $\mathrm{N}$-terminal se encuentran distribuidas irregularmente estructuras secundarias giro- $\beta$, mientras que en el dominio C-terminal predomina la estructura $\alpha$-hélice. Aunque las HMW-GS son el grupo principal de proteínas del gluten que determinan las características panaderas de la masa, las LMW-GS también cumplen un importante rol. En general, las LMW-GS se relacionan con la resistencia y la extensibilidad de la masa (Metakovskii et al., 1990; Andrews et al., 1994; Cornish et al.,
2001) e incluso algunas formas alélicas causan mayores efectos sobre las propiedades de la masa que las HMW-GS (Payne et al., 1987; Gupta et al., 1989, 1994).

Las subunidades de gluteninas de alta masa molecular (HMW-GS) presentan fuertes similitudes estructurales entre ellas, pero también diferencias características. Una de éstas es el comportamiento en la polimerización y se relaciona al número de grupos sulfhidrilo. Sobre la base de esta diferenciación, las HMW-GS son clasificadas en dos subgrupos: subunidades tipo-x y tipo-y. El dominio $\mathrm{N}$-terminal de las subunidades tipo-x contiene cuatro residuos de cisteínas y el dominio $\mathrm{N}$-terminal de las subunidades tipo-y presenta cinco residuos de cisteínas; además, las subunidades tipo-x tienen una menor movilidad electroforética que las subunidades tipo-y (Tatham, 1996).

Los estudios muestran a las subunidades de gluteninas de alto peso molecular, como moléculas alargadas que poseen una estructura $\beta$-espiral en la región central repetitiva, mientras que los dominios $\mathrm{N}$ - y C-terminal poseen una estructura similar a las proteínas globulares, presentando $\alpha$-hélices y estructura desordenada. En los dominios terminales se encuentran la mayoría de los residuos de cisteínas que proveen de los sitios para la formación de puentes disulfuro intermoleculares. La estructura $\beta$-espiral de la región central presenta elasticidad intrínseca, y aunque no se conoce en detalle su contribución a las propiedades elastoméricas del polímero, se sabe que en ausencia de agua se organiza en giro- $\beta$ (Wang et al., 2016).

\section{Estructura del gluten}

Las proteínas que integran el gluten se encuentran localizadas en cuerpos proteicos en el endosperma del grano; durante el amasado se produce la ruptura de estos cuerpos y su hidratación, formando una red tridimensional continua en donde se encuentra embebido el almidón. Las interacciones de tipo covalentes y no covalentes entre los polipéptidos más grandes que forman la masa producen una matriz elástica y extensible. El gluten es una mezcla compleja de proteínas que varían en su proporción, estructura y propiedades.

A pesar de los numerosos estudios realizados sobre la estructura de las proteínas del gluten y la base molecular existente, su rol en el procesamiento del pan no es totalmente comprendido (Shewry et al., 2001, Wang et al., 2016). Como ya se mencionó, las subunidades de gluteninas de alto peso molecular están presentes en forma de polímeros (macropolímero de gluteninas). La importancia de 
tales polímeros en la viscoelasticidad del gluten ha sido apreciada durante muchos años, aunque su tamaño, complejidad y baja solubilidad limita el estudio detallado. La importancia de los puentes disulfuro en estabilizar estos polímeros ha sido extensamente demostrada. Hay evidencia de la formación de puentes disulfuro intermoleculares entre gluteninas de alto peso molecular, y de éstas con LMW-GS (Shewry \& Tatham, 1997; Kasarda, 1999). Los enlaces disulfuro son entonces considerados esenciales para la viscoelasticidad del gluten. Sin embargo, entre las proteínas del gluten se supone que hay gran cantidad de puentes de hidrógeno debido a la alta proporción de glutamina y prolina. Otro aporte importante son las interacciones hidrofóbicas, ya que aproximadamente el 35\% de los aminoácidos poseen cadenas laterales de naturaleza apolar (Hoseney, 1994).

Las gluteninas de alto peso molecular forman la columna vertebral del polímero; estas HMW-GS se encuentran unidas entre sí a través de puentes disulfuro intermoleculares ubicados en los extremos. Esta columna vertebral de gluteninas de alto peso molecular sirve de base para que se unan a ella las subunidades de bajo peso molecular (LMW-GS) mediante puentes disulfuro formando puntos de ramificación. Las gliadinas, consideradas tradicionalmente por contribuir a la viscosidad del gluten, interactúan principalmente con los polímeros de gluteninas mediante interacciones no covalentes. Interacciones puentes de hidrógeno ocurren probablemente entre las amidas de los residuos de glutamina presentes en el dominio central HMWGS. Estas interacciones son muy importantes porque contribuyen a la viscoelasticidad de la masa (Ewart, 1989; Jeffrey \& Saenger, 1994; Belton, 1999).

Belton (1999) propuso un modelo para explicar la elasticidad del gluten. Este investigador describió al gluten formado por una estructura de bucles y colas ("loops and trains"), regiones donde existen interacciones polímero - solvente y regiones con interacciones polímero - polímero, respectivamente. En este modelo las proteínas del gluten están estabilizadas por interacciones no covalentes entre moléculas paralelas. Las HMW-GS inicialmente presentan una conformación en forma de bucle que es estirada durante la extensión del gluten para formar polímeros alineados, en los cuales la formación de altas proporciones de estructuras hoja plegada se encuentra favorecida. Por esta razón, tales polímeros son altamente resistentes a la extensión. El estiramiento de los polímeros produce, primero, la deformación de los bucles y posteriormente, el de las colas, a la vez que se rompen las interacciones no covalentes. Durante la exten- sión, los puentes disulfuro se rompen y se restablecen, derivando en una red alineada en dirección a la extensión. Sin embargo, puede producirse la restauración del equilibrio de bucles y colas espontáneamente, que resulta en la relajación de la estructura del polímero. Estos modelos estructurales fueron extensamente relacionados con la viscoelasticidad del gluten y con la estabilidad de los polímeros (Belton, 2005; Wellner et al., 2005). Shewry et al. (2003) observaron que el número y el patrón de puentes disulfuro en los polímeros de gluteninas del trigo afectan la fuerza de la masa. Por lo tanto, reafirman que las estructuras hoja plegada y los puentes disulfuro contribuyen a la estabilización de los polímeros, como fue propuesto por Belton (2005). En resumen, la calidad de una harina que permita desarrollar un gluten con buenas propiedades viscoelásticas va a depender del tipo y la cantidad de gluteninas y gliadinas que posee y de la capacidad de estas proteínas de formar enlaces intermoleculares mediante puentes disulfuro. En este sentido, promover un mayor grado de entrecruzamiento en la red polimérica podría traducirse en mejores características reológicas de las masas.

\section{Uso de enzimas para polimerizar proteínas}

Algunos autores (Gerrard et al., 1998; Gerrard et al., 2001, Huang et al., 2008) observaron que el uso de enzimas no oxidativas que incorporan entrecruzamientos entre las proteínas de la masa trigo, tales como la transglutaminasa (TG), causaban efectos positivos sobre la calidad de los productos panaderos fermentados.

En otros trabajos se estableció que la enzima TG causaba una disminución significativa del volumen específico del pan a medida que se incrementaba la dosis utilizada (Figura 1). Por ejemplo, Steffolani et al. (2010) advirtieron que cuando se utilizó $0,5 \%$ de TG, los panes desarrollaron un volumen $25 \%$ menor que el pan control (sin enzima). Mientras que, cuando la dosis utilizada fue menor $(0,01 \%)$ los panes presentaron un volumen específico similar al control. Además de la disminución del volumen de los panes debido al agregado de la enzima, también se observó que estos panes tenían un mayor peso luego de la cocción, lo que indica una menor pérdida de agua durante el horneado. El menor volumen específico del pan con el agregado de TG fue coincidente con los resultados postulados por otros autores (Basman et al., 2002; Bauer et al., 2003; Caballero et al., 2006; Caballero et al., 2007; Koh \& Ng, 2009), quienes encontraron efectos negativos de la enzima cuando las dosis 


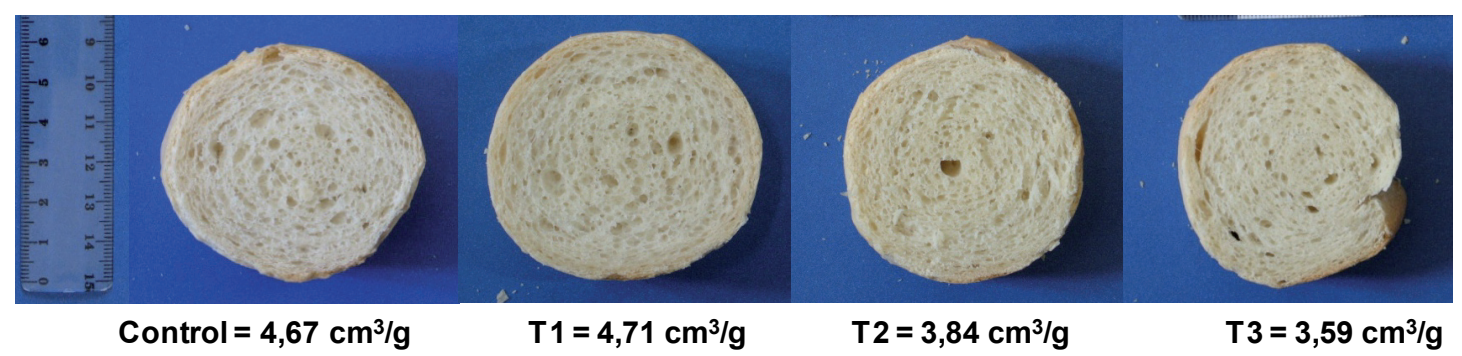

Figura 1. Fotos de panes elaborados sin enzima (control) y con 0,01\% (T1), 0,1\% (T2) y 0,5\% (T3) de transglutaminasa. Se indica el volumen específico obtenido con cada muestra

utilizadas fueron elevadas, atribuyendo este resultado al excesivo entrecruzamiento (cross-linking) que causa el endurecimiento de la masa.

Como se mencionó anteriormente, la fracción de proteínas involucrada en las reacciones de polimerización son principalmente las gluteninas (Mujoo \& Ng 2003). Larré et al. (2000) postularon que la TG convierte a las proteínas solubles en polímeros proteicos de alto peso molecular insolubles. Graveland et al. (1980) observaron que mediante el uso de detergentes (como dodecil sulfato de sodio) se podía separar una fracción de las proteínas del gluten que llamaron gel de proteínas insolubles en SDS (que más tarde fue renombrado como macropolímero de gluteninas, GMP) y concluyeron que su insolubilidad se debía principalmente al gran tamaño y al elevado grado de polimerización. Cuando se estudió el efecto de la enzima TG sobre el macropolímero de gluteninas, la enzima causó una disminución del contenido de GMP a medida que se incrementó la dosis; es decir, las proteínas del GMP fueron más solubles (Steffolani et al., 2008), contrariamente a lo observado por Larré et al. (2000). Atendiendo a los resultados contradictorios observados con respecto al efecto de la TG sobre la solubilidad del GMP, se prosiguió a confirmar la reacción de polimerización mediante un sistema modelo. Cuando la fracción de gluteninas fue incubada con TG 5\% p/p (g de transglutaminasa/100 g de gluteninas) en medio acuoso y fue seguida mediante electroforesis en geles de poliacrilamida en condiciones reductoras, se observó la formación de agregados de alto peso molecular a medida que aumentó el tiempo de incubación (Figura 2). Luego de 24 horas de incubación, la intensidad de las bandas proteicas de gluteninas comprendidas entre $31 \mathrm{KDa}$ y $45 \mathrm{KDa}$ de masa molecular (corchete rojo), y entre $66 \mathrm{KDa}$ y $200 \mathrm{KDa}$ de masa molecular (corchete amarillo) fueron significativamente menores y la intensidad de una banda proteica de masa molecular > $200 \mathrm{KDa}$ retenida al comienzo del gel separador fue significativamente mayor (Steffolani et al., 2008). En este sentido, la enzima
TG introduce nuevos enlaces peptídicos entre las gluteninas de alto peso molecular promoviendo la formación de grandes agregados solubles en SDS, por lo que promueve la formación de un gluten con una estructura de naturaleza diferente a aquella formada mediante puentes disulfuro.

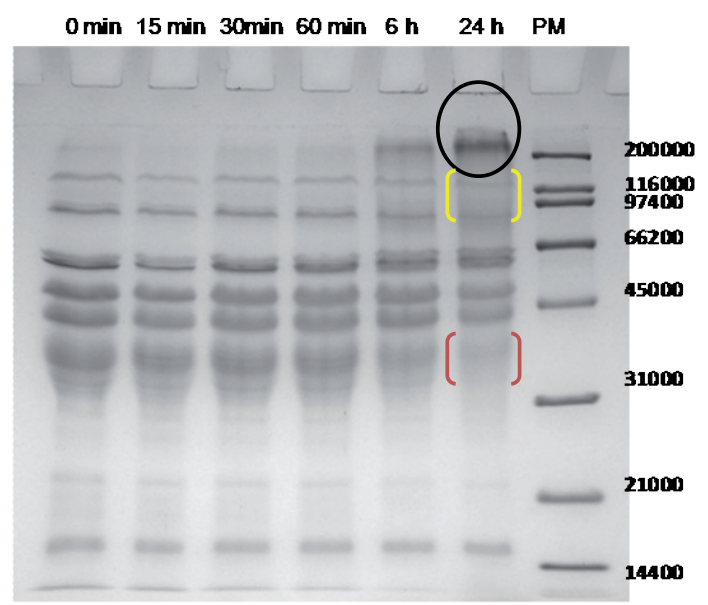

Figura 2. Incubación de gluteninas con transglutaminasa (5\% p/p). PM: Marcador de masa molecular. Tiempo de incubación: 0 , 15, 30, 60 minutos, 6 horas y 24 horas.

Con la polimerización de las proteínas del gluten claramente confirmada, se observó que este hecho no redundó en la obtención de panes de mejor calidad, por lo que es poco probable que la polimerización de otras proteínas permita hacerlo.

Esto permite concluir que es necesario trabajar sobre nuevos paradigmas, tanto para entender el rol del gluten en la panificación como en las vías para sustituirlo (Steffolani, 2010).

\section{PANES LIBRES DE GLUTEN}

Queda claro que el gluten en la harina de trigo es responsable de muchas de las características de los productos panificados, como la naturaleza viscosa y elástica necesaria para el procesamiento 
de la masa, además de proveer la matriz proteica tridimensional que impide la difusión del gas y permitir la formación y estabilización de las paredes alveolares en el pan horneado. Por este motivo, obtener un pan sin el aporte de las propiedades del gluten es un desafío considerable.

La propuesta más común para reemplazar al gluten es usar una mezcla de almidones, hidrocoloides, fibras e ingredientes lácteos para que, todos juntos, otorguen la funcionalidad necesaria. Estas materias primas tienen una mayor capacidad de absorción de agua en relación a la harina de trigo, por lo que el contenido de agua de las masas libres de gluten es generalmente alto. Esto significa que la tecnología y la metodología empleadas a la hora de obtener panes libres de gluten son distintas que las utilizadas en el proceso de panificación convencional. Las amasadoras son, en general, reemplazadas por batidoras. La masa/batido resultante se coloca en moldes y se somete a una fermentación que oscila entre los 60 y 75 minutos, y luego son horneadas por tiempos relativamente más largos, debido a la presencia de altas proporciones de agua.

En los últimos años, se han desarrollado panes libres de gluten aplicando diversos enfoques, tales como el uso de diferentes tipos de almidón (maíz, papa, mandioca o arroz), harinas de legumbres, trigo sarraceno, sorgo y granos andinos, productos lácteos, hidrocoloides, emulsificantes, proteínas sin gluten y prebióticos, para mejorar su estructura, sabor, aceptabilidad y tiempo de vida útil, como así también modificaciones en la metodología de elaboración (Arendt \& Dal Bello, 2008; Sciarini et al., 2012a; Sciarini et al., 2012b; Matos \& Rosell, 2015; Collar et al., 2015; Bourekoua et al., 2016).

Es posible encontrar en el mercado diversos panes libres de gluten; sin embargo, éstos suelen ser de una calidad pobre. Arendt et al. (2002) encontraron que la mayoría de los productos libres de gluten son de calidad inferior al ser comparados con los mismos productos a base de trigo, además de que, en general, presentan una estructura densa y seca, con un volumen bajo y una alta dureza de la miga (Gujral et al., 2003). En numerosos estudios se han investigado las propiedades de harinas de distintos cereales sin gluten (Gujral et al., 2003; Schober et al., 2005), derivados lácteos (Gallagher et al., 2003a,b), proteínas, hidrocoloides (Moore et al., 2004; Lazaridou et al., 2007) y enzimas para mejorar el volumen, la textura de la miga y la calidad global y aceptabilidad de los panes libres de gluten.

Los productos libres de gluten más difíciles de elaborar serán aquellos en los que el gluten juega un papel fundamental, es decir los que sufren un proceso de leudado. Por ello, una vía aconsejable para obtener productos de buena calidad es la obtención de una red proteica del mayor tamaño posible. Sin embargo, los resultados obtenidos con el uso de TG sobre proteínas de trigo, obligaron a ser moderados en las expectativas.

Moore et al. (2006) informaron la formación de una red proteica al incorporar huevo en polvo en formulaciones libres de gluten, luego de adicionar TG como enzima polimerizante. Pero estos autores encontraron que la formación de una red proteica antes de la incorporación de la enzima, y atribuyeron esta capacidad a las propiedades espumantes y emulsionantes de las proteínas del huevo.

La TG fue usada en harinas de arroz y en mezclas con harinas de soja, donde se comprobó su acción polimerizadora, pero el efecto sobre el volumen de pan no fue significativo (Gujral \& Rosell, 2004; Marco \& Rosell, 2008).

Estos resultados obligaron a explorar otras alternativas para la obtención de panes libres de gluten de buena calidad, ya que la idea de intentar desarrollar una red continua a partir de proteínas diferentes a las del gluten fue perdiendo fuerza con el correr del tiempo y los resultados poco satisfactorios.

Otro enfoque implica la búsqueda y exploración de diferentes mezclas de ingredientes que, juntos, otorguen funcionalidad a las masas panarias sin gluten. Almidones de distinto origen botánico pueden emplearse para mejorar la calidad de panes libres de gluten (Schober, 2009), ya que el mayor contenido de almidón significa una mayor gelatinización, lo que favorece el desarrollo de una red cohesiva que atrapa el gas y previene la pérdida de $\mathrm{CO}_{2}$ y el colapso de la corteza (Onyango et al., 2009). La harina de soja ha sido reconocida desde hace muchos años como un excelente medio para fortificar los productos a base de cereales, tales como el pan, las galletitas y las pastas. Se caracteriza por un alto contenido en proteínas ricas en aminoácidos esenciales que son fácilmente digeribles. Sin embargo, en panes de trigo, las propiedades viscoelásticas únicas del gluten se ven afectadas por la incorporación de soja. En el caso de productos de panificación libres de gluten, el efecto producido sobre la calidad tecnológica depende de los distintos tipos de harina de soja utilizados (Fleming \& Sosulski, 1978; Ribotta et al., 2004; Ribotta et al., 2005). Por ello es importante tener en cuenta que numerosos tipos de harinas son elaboradas a partir de las semillas de la soja, con propiedades muy disímiles. Dos tipos principales son: 1) harina entera, donde las legumbres son sometidas a 
un tratamiento térmico antes de ser molidas; y 2) harina deslipidizada, donde los componentes grasos son removidos por extracción con solventes, antes de la molienda. A su vez, la harina entera puede ser inactiva o activa, de acuerdo a si ha sufrido un tratamiento térmico o no, respectivamente. En la harina activa, las proteínas no se encuentran desnaturalizadas y tienen una alta solubilidad en agua fría. Esta harina posee toda la actividad enzimática presente en la legumbre; es una buena fuente de amilasas, lipasas, proteinasas, ureasas y lipoxigenasa. Por otro lado, el tratamiento térmico se realiza para optimizar el valor nutritivo de la soja, para inactivar los factores antinutricionales como el inhibidor de tripsina y reducir, además, el leve sabor amargo de la harina de soja.

Se comenzó a trabajar con la incorporación de harina de soja activa (HSA) en formulaciones de panes libres de gluten, ya que se había observado que el uso de harina sin calentar otorgaba mejores características en relación a la harina inactiva (Ribotta et al., 2004). La HSA se agregó en distintas formulaciones, mezcladas con distintas harinas y almidones, pero su efecto beneficioso fue evidente recién cuando se incorporó almidón de mandioca (Figura 3). Así, se comenzó a estudiar, desde un punto de vista fisicoquímico, si había una interacción particular que explicara el sinergismo entre ambos componentes (harina de soja activa y almidón de mandioca). Para tal fin, se trabajó con cuatro sistemas: almidón de mandioca/harina de soja activa; almidón de mandioca/harina de soja inactiva; almidón de maíz/harina de soja activa, y almidón de maíz/harina de soja inactiva. Estas mezclas fueron evaluadas en cuanto a su interacción con el agua (análisis de sinéresis), su comportamiento térmico (análisis por calorimetría diferencial de barrido -DSC), y su comportamiento reológico empírico (analizador rápido de viscosidad -RVA) y fundamental (barridos de frecuencia -reómetro oscilatorio). Además, se estudió la microestructura de las mezclas empleando un microscopio laser confocal de barrido (CLSM). Los resultados de estos análisis mostraron que el almidón de mandioca y la harina de soja activa interaccionan entre sí y que esta interacción ocurre principalmente con las proteínas de soja (el efecto de los lípidos presentes en la harina también fue estudiado, y se comprobó que su presencia o ausencia no produce cambios significativos en el comportamiento de estos sistemas). Esta interacción se verificó (i) bajo condiciones nativas (sinéresis y reología fundamental), donde las proteínas de soja pueden interactuar con el almidón, posiblemente a través de componentes asociados la superficie del gránulo de almidón, y (ii) bajo condiciones de calentamiento, donde, durante la gelatinización, el almidón de mandioca tiene un comportamiento específico, exponiendo las cadenas de amilosa/amilopectina dándoles libertad para interaccionar con las proteínas de soja, modificando los parámetros de gelatinización y, en mayor grado, los de retrogradación, ya que el efecto inhibitorio de las proteínas activas de soja sobre la retrogradación quedó también claramente comprobado. Este hallazgo es de suma utilidad para la obtención de panes libres de gluten de buena calidad y con una baja velocidad de retrogradación y por lo tanto mayor tiempo de vida útil (Sciarini et al., 2012c).

\section{CONCLUSIONES}

Del análisis de estos resultados se desprende que la mera polimerización de proteínas no es su-

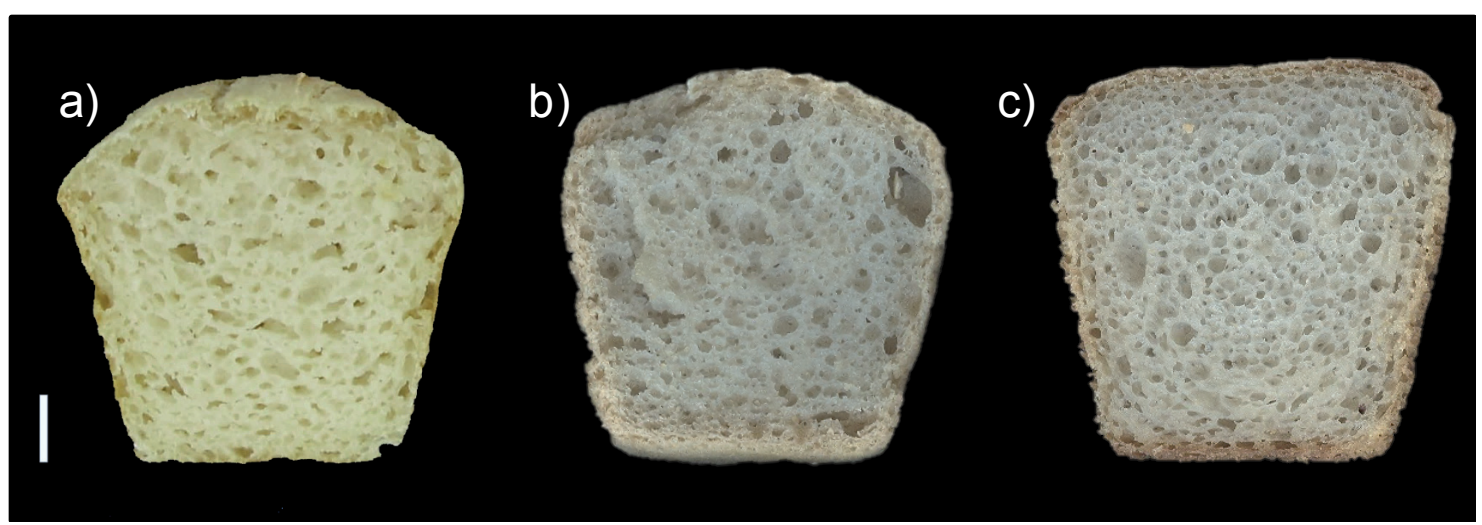

Figura 3. Panes libres de gluten elaborados a partir de diferentes formulaciones. a) Pan elaborado con harina de maíz, harina de arroz y harina de soja activa; b) Pan elaborado con harina de arroz, almidón de mandioca y harina de soja inactiva; c) Pan elaborado con harina de arroz, almidón de mandioca y harina de soja activa. Barra: $1 \mathrm{~cm}$. 
ficiente para la obtención de productos leudados libres de gluten de buena calidad tecnológica; incluso la polimerización de proteínas de trigo no conduce a una mejora de las propiedades de panes elaborados con gluten.

Las características únicas de las proteínas de trigo para la elaboración de productos leudados no son mejoradas por la polimerización, por ello, la obtención de complejos proteicos con la intención de imitar las propiedades del gluten no ha dado resultado.

La combinación de harina de soja activa con almidón de mandioca es una alternativa prometedora, ya que permite obtener productos de calidad superior. Por otra parte, el estudio de las interacciones que se establecen entre los componentes facilita la explicación de lo observado al tiempo que abre perspectivas para profundizar sus aplicaciones en nuevos desarrollos.

\section{BIBLIOGRAFÍA}

Andrews, J.L.; R.L. Hay, J.H. Skerritt and K.H. Sutton, 1994. HPLC and immunoassay based glutenin subunit analysis: screening for dough properties in wheats grown under different environmental conditions. Journal of Cereal Science 20: 203-215.

Arendt, E.K. and F. Dal Bello, 2008. Gluten-free cereal products and beverages. Academic Press, London, England. $443 \mathrm{pp}$.

Arendt, E.K.; C.M. O'Brien, T. Schober, T.R. Gormley and E. Gallagher, 2002. Development of gluten-free cereal products. Farm and Food 12: 21-27.

Basman, A.; H. Koksel and P.K.W. Ng, 2002. Effects of increasing levels of transglutaminase on the rheological properties and bread quality characteristics of two wheat flours. European Food Research Technology 215: 419-424.

Bauer, N.; P. Koehler, H. Wieser and P. Schieberle, 2003. Studies on effects of microbial tranglutaminase on gluten proteins of wheat. II. Rheological properties. Cereal Chemistry 80:787-790.

Belton, P., 1999. On the elasticity of wheat gluten. Journal of Cereal Science 29:103-107.

Belton, P.S., 2005. New approaches to study the molecular basis of the mechanical properties of gluten. Journal of Cereal Science 41: 203-211

Bloksma, A.H., 1990. Rheology of the breadmaking process. Cereal Foods World 35: 228-236.

Bourekoua H.; L. Benatallah, M.N. Zidoune and C.M. Rosell, 2016. Developing gluten free bakery improvers by hydrothermal treatment of rice and corn flours. LWT - Food Science and Technology 73: 342-350.
Caballero, P.A.; M. Gomez and C.M. Rosell, 2006. Bread quality and dough rheology of enzyme-supplemented wheat flour. European Food Research and Technology 224: 525-534.

Caballero, P.A.; M. Gomez and C.M. Rosell, 2007. Improvement of dough rheology, bread quality and bread shelf-life by enzymes combination. Journal of Food Engineering 81: 42-53.

Campbell, G.M.; C.D. Rielly, P.J. Fryer and P.A. Sadd, 1991. The measurement of bubble size distributions in an opaque food fluid. Transactions of the Institution of Chemical Engineers 69: 67-76.

Codex Alimentarius, Organización de las Naciones Unidas para la Agricultura y la Alimentación, Organización Mundial de la Salud. CODEX STAN 1181979; enmendado 1983; revisado 2008.

Collar, C.; P. Conte P, C. Fadda and A. Piga, 2015. Glutenfree dough-making of specialty breads: Significance of blended starches, flours and additives on dough behaviour. Food Science and Technology International 21:523-536.

Cornish, G.B.; F. Bekes, H.M. Allen and D.J. Martin, 2001. Flour proteins linked to quality traits in an Australian doubled haploid wheat population. Australian Journal of Agricultural Research 52: 1339-1348.

Davidson, L.S.P. and J.R. Fountain, 1950. Incidence of sprue syndrome with some observation on natural history. British Medical Journal 1:1157-1161.

Ewart, J.A.D., 1989. Hypothesis for how linear glutenin holds gas in dough. Food Chemistry 32: 135-150.

Finney, K.F. and M.A. Barmore, 1948. Loaf volume and protein content of hard winter and spring wheats. Cereal Chemistry 25: 291-312.

Fleming, S.E. and F.W. Sosulski, 1978. Microscopy evaluation of bread fortified with concentrated plant proteins. Cereal Chemistry 55: 373-382.

Gallagher, E.; T.R. Gormley and A.K. Arendt, $2003 a$. Crust and crumb characteristics of gluten free breads. Journal of Food Engineering 56: 153-161.

Gallagher, E.; A. Kunkel, T.R. Gormley and E.K. Arendt, 2003b. The effect of dairy and rice powder addition on loaf and crumb characteristics, and on shelf-life (intermediate and long term) of gluten-free breads stored in a modified atmosphere. European Food Research and Technology 218: 44-48.

Gan, Z.; R.E. Angold, M.R. Williams, P.R. Ellis, J.G Vaughan and T. Galliard, 1990. The microstructure and gas retention of bread dough. Journal of Cereal Science 12: 15-24.

Gandolfi, L.; R. Pratesi, J.C.M. Cordoba, P.L. Tauil, M. Gasparin and C. Catassi, 2000. Prevalence of celiac disease among blood donors in Brazil. American Journal of Gastroenterology 95: 689-692. 
Gerrard, J.A.; S.E. Fayle, P.A. Brown, K.H. Sutton, G.L. Simmonds and I. Rasiah, 2001. Effects of microbial transglutaminase on the wheat proteins of bread and croissant dough. Journal of Food Science 66: 782-786.

Gerrard, J.A.; S.E. Fayle, A.J. Wilson, M.P. Newberry, M. Ross and S. Kavale, 1998. Dough properties and crumb strength as affected by microbial transglutaminase. Journal of Food Science 63: 472-475.

Gomez, J.C.; G. Selvaggio, M. Viola, B. Pizarro, G. la Motta, S. de Barrio, R. Castelletto, R. Echeverría, E. Sugai, H. Vazquez, E. Mauriño and J.C. Bai, 2001. Prevalence of celiac disease in Argentina: Screening of an Adult population in La Plata area. American Journal of Gastroenterology 96:2700-2704.

Graveland, A., 1980. Extraction of wheat proteins with sodium dodecyl sulphate. Annales de Technologie Agricole 29: 113-123.

Graveland, A.; P. Bosveld, W.J. Lichtendonk and J.H.E. Moonen, 1980. Superoxide involvement in the reduction of disulfide bonds of wheat gel proteins. Biochemical and Biophysical Research Communications 93: 11891195.

Grodzinsky, E.; I. Franzen, J. Hed and M. Ström, 1992. High prevalence of celiac disease in healthy adults revealed by antigliadin antibodies. Annales Allergy 69: 66-69.

Grosch, W. and H. Wieser, 1999. Redox reactions in wheat dough as affected by ascorbic acid. Journal of Cereal Science 29: 1-16.

Gujral, H.S.; M. Haros and C.N. Rosell, 2003. Starch hydrolysing enzymes for retarding the staling for rice bread. Cereal Chemistry 80: 750-754.

Gujral, H.S. and C.M. Rosell, 2004. Functionality of rice flour modified with a microbial transglutaminase. Journal of Cereal Science 39: 225-230.

Gupta, R.B.; J.G. Paul, G.B. Cornish, G.A. Palmer, F. Bekes and A.J. Rathjen, 1994. Allelic variation at glutenin subunit and gliadin loci, Glu-1, Glu-3 and Gli1 , of common wheats. I. Its additive and interaction effects on dough properties. Journal of Cereal Science 19: 9-17.

Gupta, R.B.; N.K. Singh and K.W. Shepherd, 1989. The cumulative effect of allelic variation in LMW and HMW glutenin subunits on dough properties in the progeny of two bread wheats. Theoretical and Applied Genetics 77: 57-64.

Hoseney, R.C., 1994. Principles of Cereal Science and Technology, Second Edition. American Association of Cereal Chemists, St Paul, MN, USA, 378 pp.

Huang, W.N.; Y.L. Yuan, Y.S. Kim and O.K. Chung. 2008. Effects of Transglutaminase on Rheology, Microstructure, and Baking Properties of Frozen Dough. Cereal Chemistry 85:301-306
Janeway Jr, C.A., 2001. How the immune system works to protect the host from infection: A personal view. Proceedings of the National Academy of Sciences of the United States of America 98: 7461-7468.

Jeffrey, G.A. and W. Saenger, 1994. Hydrogen Bonding in Biological Structures, Springer-Verlag, Berlin, Germany, 569 pp.

Kasarda, D.D., 1999. Glugenin Polymers: The in Vitro to in Vivo Transition. Cereal Food World 44: 566-571.

Kent, N.L. and A.D. Evers, 1994. Bread-baking technology. En: Kent's technology of cereals. 4ta Edición. Pergamon, Oxford, Inglaterra. 191-217.

Khatkar, B.S.; R.J. Fido, A.S. Tatham and J.D. Schofield, 2002. Functional properties of wheat gliadins. II. Effects on dynamic rheological properties of wheat gluten. Journal of Cereal Science 35: 307- 313.

Koh B-K. and P.K.W. Ng, 2009. Effects of Ferulic Acid and Transglutaminase on Hard Wheat Flour Dough and Bread. Cereal Chemistry 86:18-22.

Kokelaar, J.J. and A. Prins, 1995. Surface rheological properties of bread dough components in relation to gas bubble stability. Journal of Cereal Science 22: 5361.

Kolho, K.L.; M.A. Farkkila and E. Savilahti, 1998. Undiagnosed celiac disease is common in Finnish adults. Scandinavian Journal of Gastroenterology 33:1280-1283.

Laass, M.W.; R. Schmitz, H.H. Uhlig, K.P. Zimmer, M. Thamm and S. Koletzko, 2015. The prevalence of celiac disease in children and adolescents in Germany. Deutsches Ärzteblatt International 112:553-60.

Larré, C.; S. Denery-Papini, Y. Popineau, G. Deshayes, C. Desserme and J. Lefebvre, 2000. Biochemical analysis and rheological properties of gluten modified by transglutaminase. Cereal Chemistry 77:121-127.

Larroque, O.; M. Gianibelli, M. Gómez Sánchez and F. MacRitchie, 2000. Procedure for obtaining stable extracts of cereal flour and whole meal for sizeexclusion HPLC analysis. Cereal Chemistry 77:448450.

Lazaridou, A.; D. Duta; M. Papageorgiou, N. Belc and C.G. Biliaderis, 2007. Effects of hydrocolloids on dough rheology and bread quality parameters in gluten-free formulations. Journal of Food Engineering 79: 1033-1047.

Lezcano, E., 2006. Trigo y sus derivados. Secretaría de Agricultura, Ganadería, Pesca y Alimentos. Subsecretaría de Agroindustria y Mercados. Dirección Nacional de Alimentos. Ministerio de Economía y Producción - Buenos Aires, Argentina.

Lindahl, L., 1990. Rheologycal properties in wheat flour systems. Molecular and interfacial basis. Thesis Doctoral. Lund University, Sweden. 
Ludvigsson, J.F.; D.A. Leffler, J.C. Bai, F. Biagi, A. Fasano, P.H. Green, M. Hadjivassiliou, K. Kaukinen, C.P. Kelly, J.N. Leonard, K.E. Lundin, J.A. Murray, D.S. Sanders, M.M. Walker, F. Zingone and C. Ciacci, 2013. The Oslo definitions for coeliac disease and related terms. Gut 62:43-52.

Marco, C. and C.M. Rosell, 2008. Breadmaking performance of protein enriched, gluten-free breadsEuropean Food Research and Technology 227: 1205-1213.

Matos, M.E. and C.M. Rosell, 2015. A review: Understanding gluten free dough for reaching breads with physical quality and nutritional balance. Journal of the Science of Food and Agriculture, 95: 653-661.

McRitchie, F., 2016. Seventy years of research into breadmaking quality. Journal of Cereal Science 70 : 123-131.

Medzhitov, R. and C. Janeway Jr., 2000. Innate immunity. New England Journal of Medicine 343: 338-344.

Metakovskii, E.V.; C.W. Wrigley, F. Bekes and R.B. Gupta, 1990. Gluten polypeptides as useful genetic markers of dough quality in Australian wheats. Australian Journal of Agricultural Research 41: 289-306.

Moore, M.M.; M. Heinbockel, P. Dockery, H.M. Ulmer and E.K. Arendt, 2006. Network formation in gluten-free bread with application of transglutaminase. Cereal Chemistry 83: 28-36.

Moore, M.M.; T.J. Schober, P. Dockery and E.K. Arendt, 2004. Textural comparison of gluten free and wheatbased doughs, batters and breads. Cereal Chemistry 81: 567-575.

Mora, M.; N. Litwin, M.C. Toca, M.I. Azcona, R. Solís Neffa, F. Battiston, M. Solaegui, G. Ortiz, M. Wagener, J. Olivera, S. Marchisone, G. Oropeza, C. Bastianelli, A. González and G. Rezzónico, 2012. Prevalencia de enfermedad celíaca: estudio multicéntrico en población pediátrica de cinco distritos urbanos de la Argentina. Archivo Argentino de Pediatría 110:490496.

Mujoo, R. and P.K.W. Ng, 2003. Identification of wheat protein components involved in polymer formation on incubation with transglutaminase. Cereal Chemistry 80: 703- 706.

Ng P.K.W. and W. Bushuk, 1987. Glutenin of marquish wheat as a reference for estimating molecular wheights of glutenin subunits by sodium sulfate-polyacrylamide gel electrophoresis. Cereal Chemistry 64:324-327.

Not, T.; K. Horvath, I.D. Hill, J. Partanen, A. Hammed, G. Magazzu, A. Fasano, 1998. Celiac disease risk in the USA: high prevalence of antiendomysium antibodies in healthy blood donors. Scandinavian Journal of Gastroenterology 33:494-498.

Onyango, C.; G. Unbehend and M.G. Lindhauer, 2009.
Effect of cellulose-derivatives and emulsifiers on creep-recovery and crumb properties of glutenfree bread prepared from sorghum and gelatinised cassava starch. Food Research International 42: 949955.

Osborne, T., 1907. The proteins of the Wheat Kernel. Series Carnegie Institute of Washington Publishing 84:1-119.

Payne, P.I., M.A. Nightingale, A.F. Krattiger and L.M. Holt, 1987. The relationship between HMW glutenin subunit composition and the bread-making quality in Britishgrown wheat varieties. Journal of Food Science and Agriculture 40: 51-65.

Pogna, N.; R. Redaelli, A. Dachkevitch and A. Dal Belin Peruffo, 1994. Genetics of wheat quality and its improvement by conventional and biotechnological breeding. En Bushuk W. and V. Rasper (Eds.): Wheat Production, Properties and Quality. Chapman Hall. London, England, pp 205-224.

Ribotta, P.D.; S.A. Arnulphi, A.E. León and M.C. Añón, 2005. Effect of soybean addition on the rheological properties and breadmaking quality. Journal of the Science of Food and Agriculture 85: 1889-1896.

Ribotta, P.D.; S.F. Ausar, M.H. Morcillo, G.T. Pérez, D.M. Beltramo and A.E. León, 2004. Production of glutenfree bread using soybean flour. Journal of the Science of Food and Agriculture 84: 1969-1974.

Rostami, K.; C.J. Mulder, J.M. Werre, F.R. van Brukelen, J. Kerchhaert, J.B. Crusius, A.S. Peña, F.L. Willekens and J.W. Meijer, 1999. High prevalence of celiac disease in apparently healthy blood donors suggest a high prevalence of undiagnosed celiac disease in Dutch population. Scandinavian Journal of Gastroenterology 34:276-279.

Schober, T.J., 2009. Manufacture of gluten-free specialty breads and confectionery products. En Gallagher E (Ed.): Gluten-free food science and technology. WileyBlackwell, Oxford, England, pp130-180.

Schober, T.J.; M. Messerschmidt, S.R. Bean, S-H. Park and E.K. Arendt, 2005. Gluten-free bread from sorghum: quality differences among hybrids. Cereal Chemistry 82: 394-404.

Sciarini, L.S.; G.T. Pérez, M. de Lamballerie, A.E. León and P.D. Ribotta, 2012a. Partial-Baking Process on Gluten-Free Bread: Impact of Hydrocolloid Addition. Food and Bioprocess Technology 5: 1724-1732.

Sciarini, L.S.; P.D. Ribotta, A.E. León and G.T. Pérez, $2012 b$. Incorporation of several additives into gluten free breads: Effect on dough properties and bread quality. Journal of Food Engineering 111: 590-597.

Sciarini, L.S.; P.D. Ribotta, A.E. León and G.T. Pérez, 2012c. Influence of enzyme active and inactive soy flours on cassava and corn starch properties. Starch/ Stärke 64: 126-135. 
Seguro, K.; Y. Kumazawa, C. Kuraishi, H. Sakamoto and M. Motoki, 1996. The epsilon- (gamma-glutamyl) lysine moiety in cross-linked casein is an available source of lysine for rats. Journal of Nutrition 126: 2557-2562.

Shamir, R.; A. Lerner, E. Shimar, N. Lahat, E. Sobel, R. Baror, H. Kerner and R. Eliakim, 2002. The use of a single serological marker underestimates the prevalence of celiac disease in Israel: a study of blood donors. American Journal of Gastroenterology 97:2589-2794.

Shewry, P.R. and A.S. Tatham, 1997. Disulphide Bonds in Wheat Gluten Proteins. Journal of Cereal Science 25: 207-227.

Shewry, P.R.; A.S. Tatham, J. Forde, M. Kreis and B.J. Miflin, 1986. The classification and nomenclature of wheat gluten proteins: A reassessment. Journal of Cereal Science 4: 97-106.

Shewry, P.R.; N.G. Halford and A.S. Tatham, 1992. High molecular weight subunits of wheat glutenin. Journal of Cereal Science 15: 105-120.

Shewry, P.R.; Y. Popineau, D. Lafiandra and P.S. Belton, 2001. Wheat glutenin subunits and dough elasticity: findings of the Eurowheat Project. Trend in Food Science \& Technology 11: 433 - 441

Shewry, P.R.; N.G. Halford, A.S. Tatham, Y. Popineau, D. Lafiandra and P.S. Belton, 2003. The high molecular weight subunits of wheat glutenin and their role in determining wheat processing properties. Advances in Food and Nutritional Research 45: 219- 301.

Steffolani, M.E., 2010. Efecto de las enzimas petosanasa, glucosa oxidasa y transglutaminasa en productos de panificación. Tesis doctoral Facultad de Ciencias Exactas, Universidad Nacional de La Plata. Argentina, $240 \mathrm{pp}$

Steffolani, M.E.; G.T. Pérez, P.D. Ribotta, M.C. Puppo and A.E. León, 2008. Effect of transglutaminase on the properties of glutenin macro polymer and dough rheology. Cereal Chemistry 85: 39-43

Steffolani, M.E.; P.D. Ribotta, G.T. Pérez and A.E. León, 2010. Effect of glucose oxidase, transglutaminase, and pentosanase on wheat proteins. Relationship with dough properties and bread-making quality. Journal of Cereal Science 51: 366-373.

Tatham, A., 1996. The structures of wheat protein. En Schofield J. (Ed.): Wheat Structure. Biochemistry and
Funcionality. Royal Society of Chemistry, London, England, pp 53-63.

Tatham, A.S. and P.R. Shewry, 1985. The conformation of wheat gluten proteins. The secondary structures and thermal stabilities of a-, b-, g and o-gliadins. Journal of Cereal Science 3: 104-113.

Tatham, A.S.; J.M. Field, S.J. Smith and P.R. Shewry, 1987. The conformations of wheat gluten proteins. II. Aggregated gliadins and low molecular subunits of glutenin. Journal of Cereal Science 5:203-214.

Tatham, A.S.; B. Miflin and P.R. Shewry, 1985. The Beta-Turn Conformation in Wheat Gluten Proteins: Relationship to Gluten Elasticity. Cereal Chemistry 62:405-412.

Veraverbeke, W.S. and J.A. Delcour, 2002. Wheat protein composition and properties of wheat glutenin in relation to breadmaking functionality. CRC Critical Reviews in Food Science and Nutrition 42: 179-208.

Wang, J.J.; G. Liu, Y.-B. Huang, Q.-H. Zeng, G.-S. Song, Y. Hou, L. Li and S.-Q. Hu, 2016. Role of N-terminal domain of HMW 1Dx5 in the functional and structural properties of wheat dough. Food Chemistry 213: 682690.

Weegels, P.L.; R.J. Hamer and J.D. Schofield, 1996. Functional properties of wheat glutenin. Journal of Cereal Science 23: 1-18.

Wellner, N.; E.N. Mills, G. Brownsey, R.H. Wilson, N. Brown, J. Freeman, N.G. Halford, P.R. Shewry and P.S. Belton, 2005. Changes in protein secondary structure during gluten deformation studied by dynamic Fourier transform infrared spectroscopy. Biomacromolecules 6: 255-261.

Wieser, H., 2003. The use of redox agents. En Cauvain, S.P. (Ed.): Bread Making; Improving Quality. Woodhead Publishing Ltd., Cambridge, UK, pp. 424-446.

Wieser, H., 2007. Chemistry of gluten proteins. Food Microbiology 24:115-9.

Wrigley, C.; J. Andrews, F. Bekes, P. Gras , R. Gupta, F. MacRitchie and J. Skerritt, 1998. Protein-protein interactions-essential to dough rheology. En Hamer R. and R.J. Hoseney (Eds.): Interactions: The Keys to Cereal Quality. American Association of Cereal Chemists, St. Paul, MN, USA, pp 18-20. 